Military Technical College Kobry El-Kobbah, Cairo, Egypt

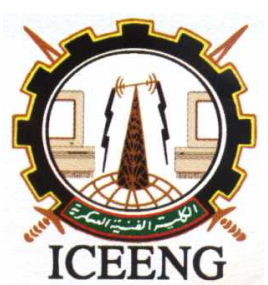

\section{$6^{\text {th }}$ International Conference on Electrical Engineering ICEENG 2008}

\title{
Frequency Hopping Overcomes Multipath Fading using FEC
}

By

Muhammad Asif *

\author{
Othman Sidek ** \\ Prof. Farid Ghani *** \\ Umair Soori****
}

\section{$\underline{\text { Abstract: }}$}

In this paper we employ the codes with shorter length, which are chained with each other in such a way to bring forth the multilevel coding while using Reed Solomon (RS) decoding as forward error correction (FEC). The dependably of these chained codes can be ascertained from the simulation results especially when the system is under the menace of hostile jammers and interference, which turn out to be reduced number of errors. Fast frequency hopping (FFH) and multicarrier transmission are the diversity techniques used in this paper as conventional and alternative techniques respectively for the system.

\section{Keywords:}

Reed Solomon, Frequency hopping spread spectrum and Multipath fading

* FAST - National University of Computer and Emerging Sciences, Karachi 75030, Pakistan

** Collaborative MicroElectronic Design Excellence Center (CEDEC) Universiti Sains Malaysia, Engineering, Pulau Pinang, Malaysia

*** School of Computer and Communication Engineering Universiti Malaysia

**** School of Electrical and Electronic Engineering, Universiti Sains Malaysia 


\section{Introduction:}

A physical channel with a bombastic assortment is feasible for the digital modulation and demodulation of the information carrier over an additive Gaussian noise channel (AWGN). In the last 20 years, our daily life is dominated by the applications of wireless communications which cannot be abnegated being as an essential part of our requisite. The excessiveness of eminent and reliable band of wireless services bob up with the new challenges for application and design engineers, not only to provide the better services, also need to heighten the current system to next generation of wireless communications. The wireless communication systems are confronting a problem of fading which affects the communication signal. To get over this problem diversity and channel coding techniques has been introduced.

The combination of frequency hopping spread spectrum (FHSS) with Reed Solomon coding has been adopted by many systems employing PSK modulation, easy for the frequency synthesizer to maintain the phase coherence. But in this paper we have employed fast frequency hopping (FFH) spread spectrum with Reed Solomon coding, where PSK modulation fails to maintain the coherence so the FSK non-coherent modulation has been introduced which get over the desire result successfully.

\subsection{Frequency Hopping Spread Spectrum (FHSS) with Reed Solomon coding:}

In FHSS systems, the pseudorandom defined frequency carrier hopped the modulated symbols, which are dehopped synchronously at the receiver end.

It is long-familiar that the transmission of a code symbol over many different hops, chained with a forward error correcting code is an efficient means of evading the partial band noise jammer in a frequency hopping spread spectrum communications system. A lot of efficacious methods are assumed for combining the diversity of transmission. [1-4].

\subsection{Reed Solomon codes:}

The omnipresence and usefulness of Reed-Solomon codes are the core virtues which work effectively in the field of wireless communication.

Reed-Solomon is successfully positioned in all ongoing investigations in operation and has booming usage in future tasks. In deep space communication system ReedSolomon codes, chained with convolutional codes, encounter critical role.

Reed-Solomon codes were used in the 2004 Mars Exploration Rover mission, in order to explore surface and geology of Martian. For digital video broadcasting the 
services of conventional Reed-Solomon codes and chained Reed-Solomon codes are used to reduce the number of errors. In the third generation $(3 \mathrm{G})$ wireless standard, such as CDMA2000, the outer codes of Reed-Solomon codes have been employed due to its successful deportment as FEC.

\section{Multipath Fading:}

In wireless communication system we usually take multipath fading channels as a touchstone channel model. Other than the bandwidth restraints it is not cumbersome to find the channel capacity of multipath fading channels, in order to keep the rates under capacity, the transmission bandwidth must be made indiscriminately large for the small probability of error which has been presented in [5].

From the aforementioned discussions we come to the point that it is the transmission bandwidth, not the decoding delay, which decreases the number of errors by increasing the bandwidth.

A signal fading adds up into existence when unlike carrier and phase offsets add annihilatively at one time. Time variation acts an authoritative role in the structure of the radio channels medium. The response of channel is highly affected due to variation in time and the physical change in the medium of channel alters the received signal and the users fail to achieve the coveted result. Equation (1) shows the unmodulated signal carrier which portrays the time variant multipath channel.

$c(t)=A \cos 2 \pi f_{c} t$

When there is no sign of noise the received signal is expressed as

$x(t)=A \sum_{n} \alpha_{n}(t) \cos \left[2 \pi f_{c}\left(t-\tau_{n}(t)\right)\right]$

where $\alpha_{n}(t)$ is attenuation factor of time variant assorted with the $n^{\text {th }}$ path and the proportionate delay is represented by $\tau_{n}$.

\section{System Model:}

The block diagram of FHSS is depicted in figure 1 with forward error decoder and Pseudonoise generator for pseudorandom codes. At the receiver, the frequency synthesizer synchronized the frequency pattern produced at transmitter end. The 
mixer successfully dispatches the pattern from the received signals. A band pass filter present at the output of mixer filters the signal and acquires the dehopped demodulated signal.

The dehopped output is comprised of $L$ diversity for $n$ code of symbols. These $L$ diversity receptions are employed in the side information in order to create and build the new statistic decision irrespective of the presence of interference and jammer threats.

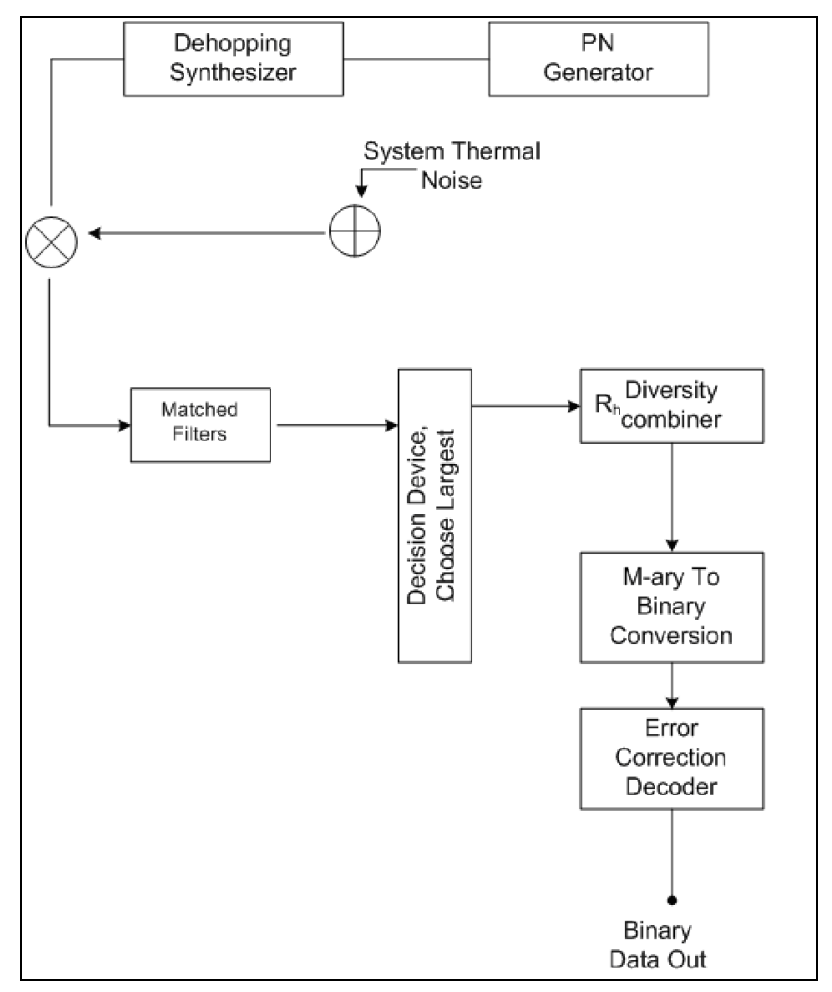

Figure (1): Block diagram of frequency hopping spread spectrum with Reed Solomon encoder

\section{Results and Discussion:}

The approach of fast frequency spread spectrum (FFHSS) is acquired to overcome the multipath fading for digital transmission. The transmission of a code symbol over many different hops caters protection against the intentional and unintentional interference. Fast frequency hopping (FFH) and multicarrier transmission are the diversity techniques used in FHSS system as conventional and alternative techniques respectively for the system. 
The original which has been generated as shown in figure 2 is passed through multiple recognitions of the signal complex envelope with stochastic phases without FEC which is portrayed in figure 3. The simulated result obtains in figure 4 is give for comparison of uncoded transmission signal at the same spectral efficiency. While figure 5 shows the compatibility of the system in noisy environment which shows the system corresponding to FEC achieves the best performance when using erasure RS decoding. The Probability of error for coded system is depicted in figure 6, while the eye diagram of the coded system shows the compatibility of system while using FHSS in fading environment. 


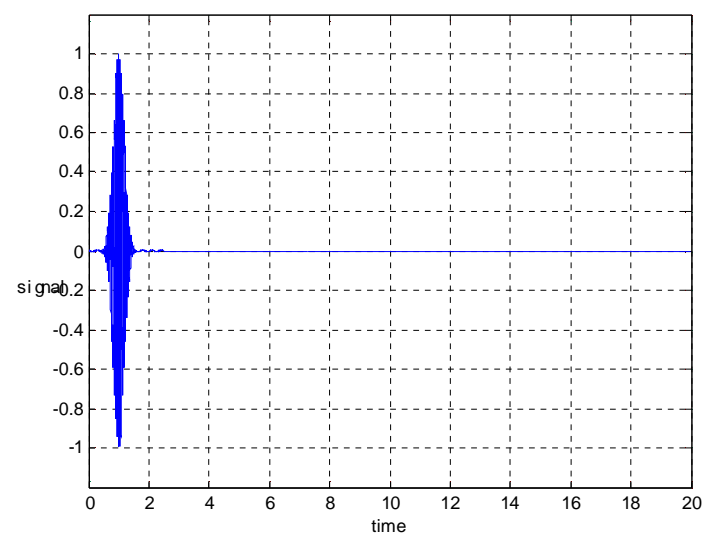

Figure (1): Real Signal Envelope

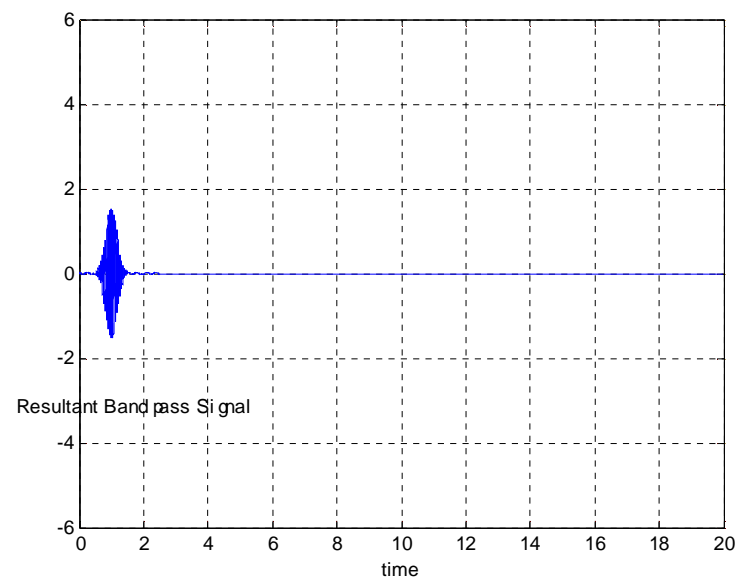

Figure (2): Resulting complex signal envelope

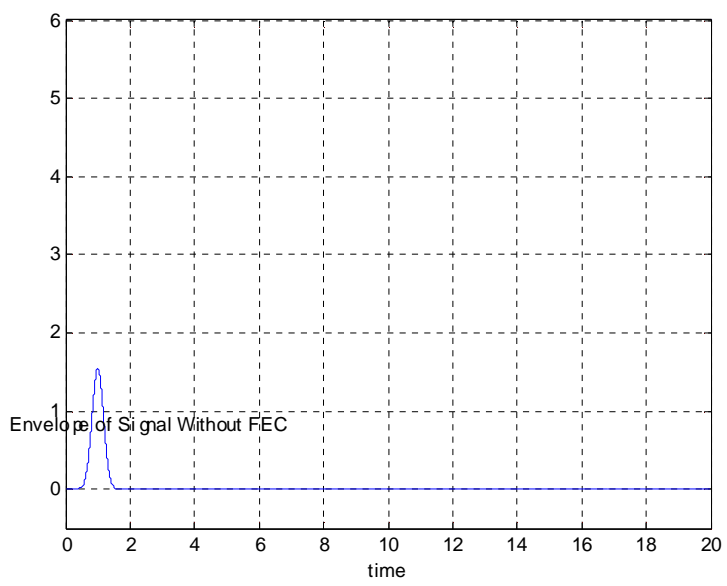

Figure (3): Generation of signal without Forward Error Code 


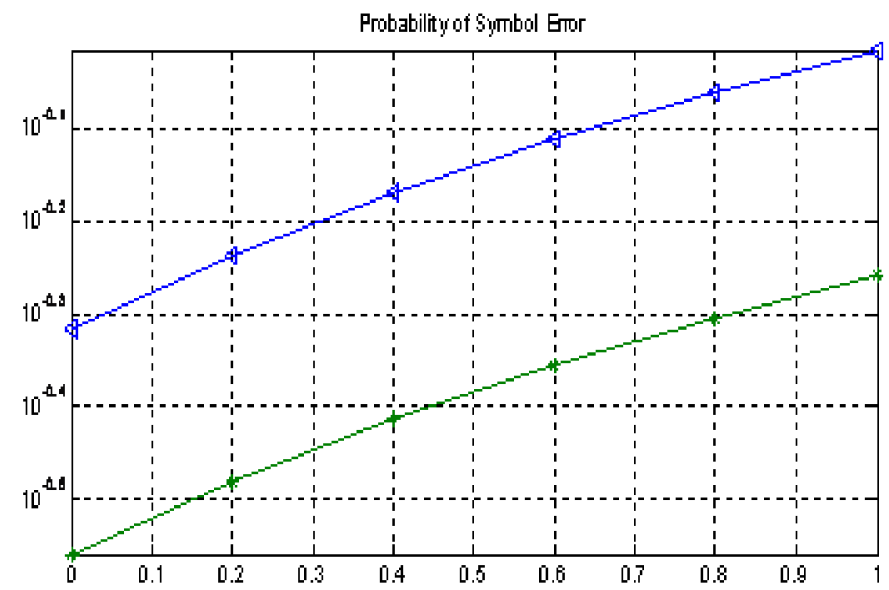

Figure (5): Probability of symbol error for coding system

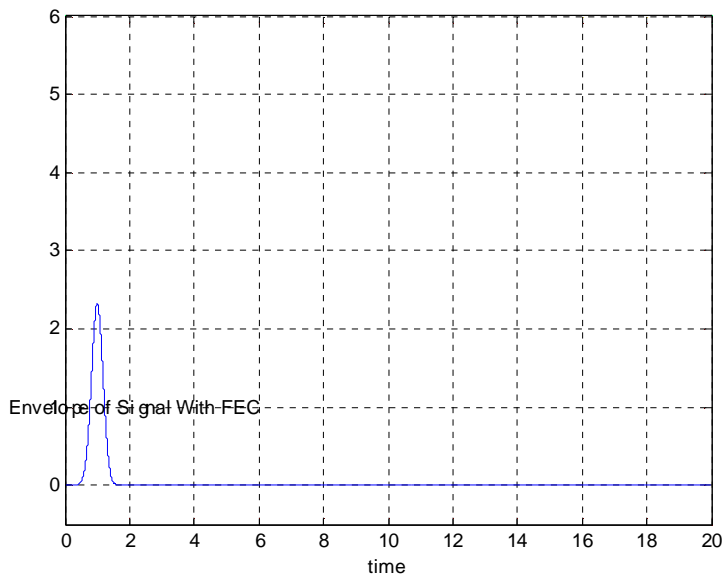

Figure (4): Generation of signal with Forward Error Code 

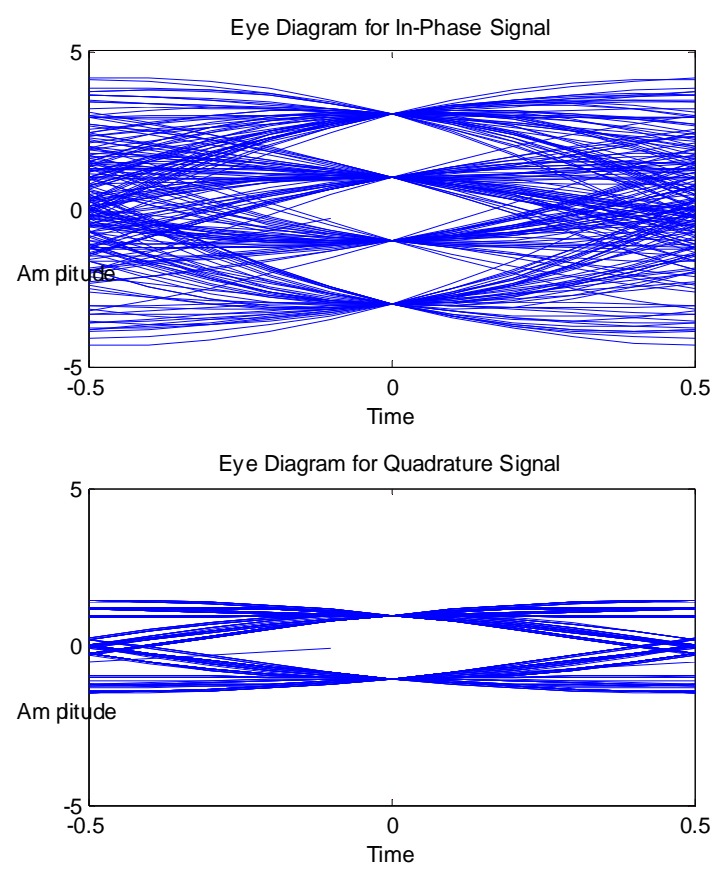

Figure (6): Eye Diagram of the signal after

\section{Conclusions:}

In this paper we employ the codes with shorter length, which are chained with each other in such a way to bring forth the multilevel coding while using Reed Solomon (RS) decoding as forward error correction (FEC). The dependably of these chained codes can be ascertained from the simulation results especially when the system is under the threat of hostile jammers and interference. The approach of fast frequency spread spectrum (FFHSS) is acquired to overcome the multipath fading for digital transmission. The transmission of a code symbol over many different hops caters protection against the intentional and unintentional interference.

\section{References:}


[1] W. E. Stark, "Coding for frequency-hopped spread-spectrum communication with partial-band interference-Part 11," IEEE. Trans. Commun., , pp. 10451057, 1985.

[2] B. K. Levitt, J. K. Omura, and R. J. Stokey, "Coding tradeoffs for improved performance of FH-MFSK systems in partial band noise," in Proc. NTC81, 1981, pp. D9.1.1-D9.1.5.

[3] L. E. Miller, J. S. Lee, and A. P. Kadrichu, "Probability of error analysis of a BFSK frequency hopping system with diversity under partial-band jamming interference-Part 111: Performance of a square- law self normalizing soft decision receiver," IEEE Trans. Commun., vol.(34), pp. 669-675, July 1986.

[4] M. K. Simon, J. K. Omura, R. A. Scholtz, and B. K. Levitt, Spread Spectrum Communications vol ( I). Rockville, MD: Computer Science, 1985

[5] T. Emre and N.C. David. "Capacity and mutual information of wideband multipath fading channels." IEEE Trans. Inform. Theory, 46(4):1384-1400, 2000. 